\title{
Un niño de 9 años de edad con una placa de aspecto alopécico y problemas familiares
}

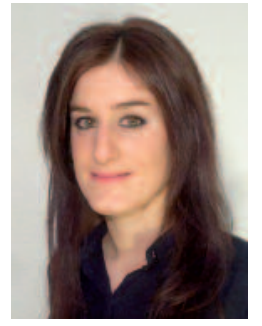

Sara Burillo Martínez

Médico interno residente de Dermatología.

Hospital Universitario 12 de Octubre. Madrid.
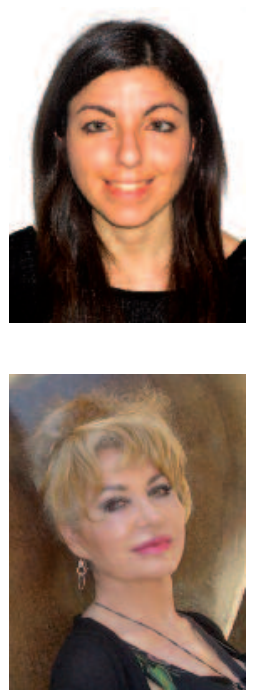

Fátima Tous Romero

Médico interno residente de Dermatología.

Hospital Universitario 12 de Octubre. Madrid.

\section{Aurora Guerra Tapia}

Doctora en Medicina.

Jefa de Sección de Dermatología. Hospital Universitario 12 de Octubre. Madrid.

Profesora titular de Dermatología Médico-quirúrgica y Venereología. Universidad Complutense de Madrid. Profesora de Dermatología. Universidad Internacional de La Rioja. Logroño (La Rioja).
Mi paciente es un niño de 9 años de edad que acudió a la consulta acompañado por su padre. Al entrar en la consulta, nos llamó la atención su aspecto triste, baja talla y bajo peso para su edad. Había sido derivado por su pediatra para que valorásemos una extensa placa de aspecto alopécico en el área interparietal del cuero cabelludo, que había aparecido hacía tres meses. A la exploración física, la placa presentaba cabellos de distinta longitud, bordes geométricos que le proporcionaban un aspecto artificial, ningún signo de inflamación o atrofia y maniobra de pilotracción negativa (fig. 1). La tricoscopia comprobó cabellos cadavéricos entre los normales. El resto de la exploración física resultó anodina, salvo, como hemos citado previamente, la baja talla y el bajo peso para su edad.

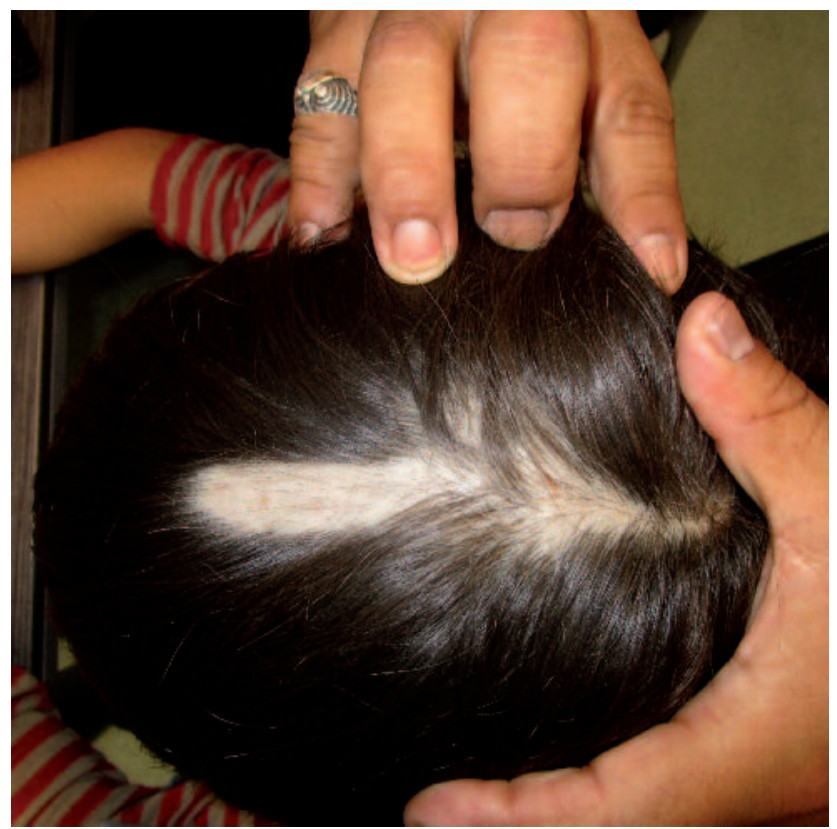

Figura 1. Placa de tricotilomanía. Obsérvense las manos del padre que dan información trascendental sobre el entorno. 
Con el diagnóstico de tricotilomanía, iniciamos el tratamiento con $\mathrm{N}$-acetilcisteína, junto a un nutricosmético coadyuvante en los tratamientos de alopecia en general que contuviese elementos adecuados como cistina, biotina y hierro, y explicamos al padre la naturaleza de la lesión. En la revisión, confirma que ha observado a su hijo traccionar el cabello y que se ha documentado en Internet, comprobando que las fotografías son iguales a las de su hijo. Explica que viven en la calle tras haber sido desahuciados. Ante tan llamativo modo de expresar su hijo su descontento, el padre reaccionó y finalmente consiguió acceder a una vivienda de protección oficial. La recuperación fue completa y dimos de alta a un paciente de aspecto mucho más alegre.

Casualmente, poco después, acudió a la consulta otro paciente, en este caso, una niña de 10 años de edad acompañada por su abuelo, para que valorásemos una placa «alopécica» de reciente aparición de características muy similares a la del paciente anterior (fig. 2).

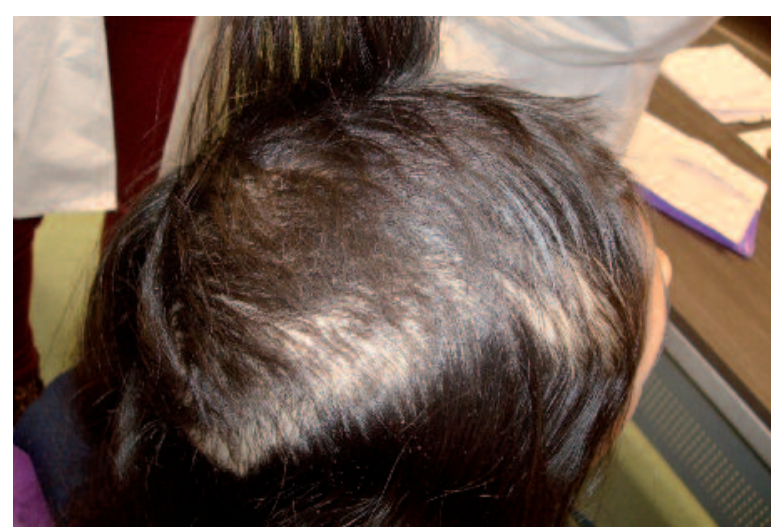

Figura 2. Placa de tricotilomanía en el área interparietal del cuero cabelludo. Se observa el largo cabello de las zonas no traccionadas.

Igualmente, nuestro diagnóstico fue el de tricotilomanía e iniciamos el tratamiento con $\mathrm{N}$-acetilcisteína, explicando al abuelo en esta primera consulta que el proceso «tiene relación con los nervios». En la revisión, el abuelo muestra su rechazo al diagnóstico y se enfrenta al equipo médico con insultos basados en su incredulidad del diagnóstico, ante lo que recomendamos, con paciencia, documentarse sobre la enfermedad. Por otra parte, investigamos por qué no han acudido los progenitores y el abuelo explica que tienen otro hijo gravemente enfermo que precisa cuidados constantes: recomendamos que acudan en adelante. En la siguiente revisión, a la que acuden padres y abuelo, quien se disculpa, la paciente admite el hábito de traccionarse el cabello. La llamada de atención de la paciente provoca un cambio de actitud en los progenitores, produciéndose su recuperación total. No se han dado recidivas a lo largo de más de un año de seguimiento, con revisiones periódicas a las que, desde el diagnóstico, también acuden los progenitores.

La tricotilomanía es un trastorno del control de los impulsos consistente en el arrancamiento del pelo ${ }^{1,2}$. Si bien ya encontramos referencias en la Biblia o en la obra de William Shakespeare, en la literatura médica, fue descrita por primera vez en 1889 por el dermatólogo francés François Henri Hallopeau, quien acuña el término tricotilomanía para describir la irresistible necesidad de sus pacientes de traccionar el pelo ${ }^{3}$.

La alopecia resultante puede acarrear un importante impacto negativo en el funcionamiento psicosocial de los pacientes, pero, además, la tracción recurrente de los cabellos puede producir un daño permanente en el folículo piloso, desembocando en una alopecia cicatricial irreversible $\mathrm{e}^{4,5}$.

En la infancia, la tricotilomanía es, en muchas ocasiones, reflejo de problemas en el núcleo familiar, por lo que la intervención en el entorno es clave en el tratamiento ${ }^{2}$. En el primer caso, no representa sino un modo de expresar el paciente su descontento ante una situación social desfavorable en el contexto actual de recesión económica que viven hoy muchas familias. En el caso de la segunda paciente, una llamada de atención, reclamando un cambio de actitud en unos progenitores tan ocupados en el cuidado constante de su otro hijo enfermo. Por lo tanto, la interven- 
ción en el entorno es fundamental en el tratamiento y, así, en nuestros dos pacientes, la recuperación fue ad integrum tras la aceptación del diagnóstico por los progenitores y la modificación de sus conductas. Aunque hemos de decir que, en muchas ocasiones, la actitud inicial por parte de la familia es de rechazo al diagnóstico y al equipo médico, como ocurrió con el abuelo de la segunda paciente.

Por otra parte, creemos que el tratamiento con $\mathrm{N}$-acetilcisteína, un modulador del glutamato extracelular, es necesario no solo por su papel neuromodulador $^{6,7}$, sino también por constituir por sí mismo un motivo de atención hacia los pacientes que, como ejemplariza el segundo caso, es muchas veces lo demandado por el enfermo.

Los nutricosméticos aceptados para el tratamiento de la alopecia en general cumplen en este proceso, a menudo, un papel coadyuvante, ya que, por una parte, el paciente siente que está siendo tratado con un producto farmacológico específico para la alopecia, lo que aumenta su confianza en el tratamiento, y, por otra, se está facilitando al pelo en crecimiento los elementos imprescindibles para su correcto desarrollo ${ }^{8}$.
Aportamos dos casos de tricotilomanía en edad pediátrica como reflejo de dificultades en el núcleo familiar. El entorno familiar es fundamental en el desencadenamiento de la tricotilomanía y en la evolución favorable cuando se modifica.

\section{BIBLIOGRAFÍA}

1. Millard LG, Millard J. Trichotillomania. En: Burns T, Breathnach S, Cox N, Griffiths C (eds.). Rook's Textbook of Dermatology. 8. ${ }^{\text {a }}$ ed. Oxford: Wiley-Blackwell; 2010. p. 64.30-64.33.

2. Rodríguez Pichardo A, García Bustínduy MC. Tricotilomanía. En: Guerra Tapia A (ed.). Dermatología psiquiátrica, Volumen I: De la mente a la piel. Barcelona: Editorial Glosa; 2008. p. 47-62.

3. Hallopeau H. Alopécie par grattage (trichomanie ou trichotillomanie). Ann Dermatol Syphil. 1889;10:440-1.

4. Huynh M, Gavino AC, Magid M. Trichotillomania. Semin Cutan Med Surg. 2013;32(2):88-94

5. Harrison JP, Franklin ME. Pediatric trichotillomania. Curr Psychiatry Rep. 2012;14(3):188-96.

6. Grant JE, Odlaug BL, Kim SW. N-acetylcysteine, a glutamate modulator, in the treatment of trichotillomania: a doubleblind, placebo-controlled study. Arch Gen Psychiatry. 2009; 66(7):756-63.

7. Rothbart R, Amos T, Siegfried N, Ipser JC, Fineberg N, Chamberlain SR, et al. Pharmacotherapy for trichotillomania. Cochrane Database Syst Rev. 2013;11:CD007662.

8. Guerra Tapia A. Inhibidores de la 5 alfa reductasa en la alopecia androgénica: presente y futuro. Opciones terapéuticas. Act Dermatol. 2007;46(8-9):539-47. 